\title{
A CURIOUS IRREDUCIBLE CONTINUUM
}

\author{
BY W. A. WILSON
}

One of Janiszewski's theorems* is to the effect that, if $a b$ is a bounded irreducible continuum and $c$ is a point of the first species, there is a decomposition of $a b$ into two continua, $a c$ and $c b$, such that $a c \cdot c b=c$. The fact that the condition imposed on $c$ is not a necessary one naturally led to the question as to whether an irreducible continuum not made up of indecomposable continua can always be decomposed into two continua having only one point in common. A vain endeavor to answer this question in the affirmative resulted in the following example to the contrary, which may be of interest to workers in this field.

Although the continuum is simple, it is difficult to describe without a figure and therefore the construction should be carried out through the first two stages.

The first stage in the construction is as follows. Take a unit square in the first quadrant and mark the following points: $\quad a=(0,0), \quad b=(1 / 4,0), \quad c=(1 / 2,0), \quad d=(3 / 4,0)$, $e=(1,0), \quad f=(1 / 2,1 / 4), \quad g=(3 / 4,1 / 4), \quad h=(1 / 4,3 / 4), \quad i=$ $(1 / 2,3 / 4), j=(0,1), k=(1 / 4,1), \quad l=(1 / 2,1), \quad m=(3 / 4,1)$, $n=(1,1)$. Draw the straight lines $b k, c f, f h, g i, i l$, and $d m$. Set $E_{1}=b k+c f+f h$ and $E_{2}=g i+i l+d m$. The continuum $E_{1}$ may be regarded as the union of two continua, $b k$ and $c f$ $+f h+h k$, extending from $a e$ to $j n$ and having a common segment $h k$ of length $1 / 4$. A similar statement holds for $E_{2}$. The continua $E_{1}$ and $E_{2}$ divide the square into five "strips," of which two, $b c f h$ and ligm, meet only one side of the square and are called incomplete. The other three extend from $a e$ to $j n$ and are called complete; two of them are rectangles, $R_{1}=a b k j$ and $R_{2}=d e n m$, and the third is a polygon $P_{1}=$

* Z. Janiszewski, Sur les continus irréductibles entre deux points, JouRnaL de l'École Polytechnique, (2), vol. 16 (1926), p. 125. 
cdgilkhf; all three have a uniform width of $1 / 4$ measured parallel to the $x$-axis.

The first step in the second stage is to repeat this construction in the rectangle $R_{1}$ with certain modifications. The points corresponding to $b, c, d$, etc., are : $b^{\prime}=(1 / 16,0)$, $c^{\prime}=(1 / 8,0), d^{\prime}=(3 / 16,0), f^{\prime}=(1 / 8,1 / 8), g^{\prime}=(3 / 16,1 / 8)$, $h^{\prime}=(1 / 16,7 / 8), \quad i^{\prime}=(1 / 8,7 / 8), \quad k^{\prime}=(1 / 16,1), l^{\prime}=(1 / 8,1)$, $m^{\prime}=(3 / 16,1)$. The essential thing to notice is that the segments $c^{\prime} f^{\prime}, d^{\prime} g^{\prime}, h^{\prime} k^{\prime}$, and $i^{\prime} l^{\prime}$ each have a length equal to one-half the length of the corresponding segments $c f$, $d g, h k$, and $i l$. We now have two new continua $E_{3}$ and $E_{4}$ which have the same properties as $E_{1}$ and $E_{2}$ except that the common segments $h^{\prime} k^{\prime}$ and $d^{\prime} g^{\prime}$ have a length of $\frac{1}{2} h k=\frac{1}{8}$. They divide $R_{1}$ into five strips, of which three are complete and have a uniform width of $1 / 16$.

If each point $(x, y)$ of $E_{3}$ and $E_{4}$ is moved to the position $\left(x^{\prime}, y^{\prime}\right)$ defined by $x^{\prime}=x+1 / 4$ if $y \geqq 3 / 4, x^{\prime}=x+1 / 4+\frac{1}{2}\left(\frac{3}{4}-y\right)$ if $1 / 4 \leqq y \leqq 3 / 4, x^{\prime}=x+1 / 2$ if $y \leqq 1 / 4$, and $y^{\prime}=y$, the images $E_{5}$ and $E_{6}$ of $E_{3}$ and $E_{4}$ will be two continua dividing $P_{1}$ exactly as $E_{3}$ and $E_{4}$ divide $R_{1}$. Likewise the transformation $x^{\prime}=x+3 / 4, y^{\prime}=y$ gives two continua $E_{7}$ and $E_{8}$ dividing $R_{2}$. Each of the continua $E_{5}, E_{6}, E_{7}$, and $E_{8}$ is the union of two lines or broken lines extending from $a e$ to $j n$ and having a common segment of length $1 / 8$. We now have eight continua, $E_{1}, E_{2}, \cdots, E_{8}$, which define nine complete strips, each of width $1 / 4 \times 1 / 4=1 / 16$.

Now repeat the construction of the second stage in these nine strips, again making the segments corresponding to $c^{\prime} f^{\prime}, d^{\prime} g^{\prime}$, etc., one-half as long as before. This will give us 18 more continua, $E_{9}, E_{10}, \cdots, E_{26}$, each of which is the union of two lines or broken lines extending from ae to $j n$ and having a common segment of length $1 / 2 \times 1 / 8=1 / 16$. These divide the nine complete strips of the second stage into 18 incomplete strips and 27 complete strips, each of the latter having a width of $1 / 4 \times 1 / 16=1 / 64$.

If this process is carried on indefinitely, the width of the complete strips converges to zero and we get an enumer- 
able set of continua $E_{n}$ of the type described above, whose common segments have a length converging to zero as $n$ increases. If $B$ is the sum of the sets $E_{n}, \bar{B}$ is the desired continuum $C$. The improper limiting points of $B$ constitute a non-enumerable set of simple arcs joining $a e$ and $j n$. Thus $C$ is the sum of an infinity of continua joining $a e$ and $j n$, which we may call the elements of $C$. We distinguish these by calling the sets $E_{n}$ elements of the first class, and the simple arcs elements of the second class.

That $C$ is a continuum and is irreducible between any point $\alpha$ of $a j$ and any point $\beta$ of $e n$ is easily deduced from well known theorems. The proof that $C$ cannot be expressed as the union of two continua $C_{1}$ and $C_{2}$, which have but a single point in common, is briefly as follows. Neither $C_{1}$ nor $C_{2}$ contains points of both $a j$ and $e n$; let $a j$ be a part of $C_{1}$ and let $e n$ be a part of $C_{2}$. Let $x$ be a point of $C_{1} \cdot C_{2}$ and lie on an element $F$ of $C$. Then $C_{1}$ contains all the elements at the left of $F$ and $C_{2}$ contains all those at the right; let $C_{1}{ }^{*}$ denote the sum of the first set and $C_{2}{ }^{*}$ that of the second. If $F$ is one of the elements $E_{n}$, one of the two continua forming $E_{n}$ lies in $\bar{C}_{1}{ }^{*}$ and the other in $\bar{C}_{2}{ }^{*}$. But these have a common segment of length greater than zero; hence $C_{1} \cdot C_{2}$ contains a continuum which is not a point. If $F$ is an element of the second class, $F$ lies in both $\vec{C}_{1}{ }^{*}$ and $\bar{C}_{2}{ }^{*}$, and hence in $C_{1} \cdot C_{2}$. Thus $C$ cannot be expressed as the union of two continua which do not have at least one continuum which is not a point in common.

YALE UNIVERSITY 